\title{
Normalization of the a1-Na/K-ATPase/Src Signalosome Restored Microbiota Communities and Rescinded NASH Progression in the Mouse
}

Schade $\mathbf{M}^{2-3}$, Sanabria JA ${ }^{1-2}$, Mallick $A^{1-2}$, Denvir, J. ${ }^{3}$, Sanabria JD ${ }^{1-2}$, Aguilar $\mathrm{R}^{1-2}$, Andryka $\mathrm{M}^{1-2}$, Schlatzer $\mathrm{D}^{5}$, Li $\mathrm{X}^{5}$, Hazlett FE ${ }^{6}$, Kachman $\mathrm{M}^{6}$, Raskind $\mathrm{A}^{6}$, Udoh UA ${ }^{1-2}$, Rajan $\mathrm{PK}^{1-2}$, Sanabria JD'-2, Brunengraber $\mathrm{H}^{4}$, Sodhi $\mathrm{K}^{1-3}$, Pierre $\mathrm{SV}^{2}$, Xie $\mathrm{Z}^{2-3}$, Shapiro $\mathrm{Jl}^{2-3}$, and Sanabria $\mathrm{JR}^{1-2,4-5}$

${ }^{1}$ Department of Surgery and ${ }^{2}$ Marshall Institute for Interdisciplinary Research (MIIR)

${ }^{3}$ Marshall University Genomics Core Facility, ${ }^{4}$ Marshall University Joan Edwards School of Medicine, Huntington, ${ }^{5}$ Department of Nutrition and Metabolomic Core Facility, Case Western

Reserve University School of Medicine, Cleveland, $\mathrm{OH}$, and ${ }^{6}$ Metabolomic Core Facility, University of Michigan, Ann Arbor, MI

Corresponding author: J Sanabria MD MSc

Email: sanabriaj@marshall.edu

juan.sanabria@case.edu

Running head: Na/K-ATPase signaling in NASH progression

Keywords: pSrc, Glutathione, Na/K-ATPase, metabolism, metabolic prints, metabolomics, rodent, NAFLD, NASH, high-fat diet, fibrosis, inflammation, senescence

\section{Authors Contributions:}

Design and animal work: SM, SJA, BH, SJ

Laboratory work: SM, SJA, MA, DJ, SJD, SD, LX, HFE, KM, UUA, RPK

Gathering of data and statistical analyses: SM, SJA, RA, SJD, AM, MA, SJ, KM, RA, SJD

Writing and editing SM, SJA, SD, LX, BH, PS, SK, UUA, XZ, SJD, SJI, SJ

Manuscript text word count:

Number of figures: 5

Tables: 0

Number of web-links: 1

Supplemental material: 1 (Text plus 5 Figures and 1 Table)

Financial support: MUSOM, JE Comprehensive Cancer Center Foundation, and NIH WVINBRE grant P20GM103434

Abbreviations: aa: amino-acid; ANOVA: analysis of variance; ESLD: end-stage liver disease; GSH: glutathione reduced; GS:SG: glutathione oxidized; $H A V, H B V$, and $H C V$ : hepatitis $A$, B, and $C$ viral infection, respectively; HCC: hepatocellular carcinoma; HFD: high-fat diet; HTN: hypertension; IVC: infra-hepatic vena cava; IP: intraperitoneal; MRI: magnetic resonance imaging; NAFLD: non-alcoholic fatty liver disease; NASH: non-alcoholic steatohepatitis; NKA: $\mathrm{Na} / \mathrm{K}-\mathrm{ATPase}$ NMC: normal mouse chow; NS: normal saline $0.9 \%$; LC-MS: liquid chromatography mass-spectrometry; PNK: pNaKtide; RT-PCR: real time-polymerase chain 
Schade \& Sanabria et al. NKA/Src in NASH progression

reaction; PCA: principal component analysis; Src: sarcoma kinase; $T B W$ : total body weight; $W B$ : Western blot.

\section{ABSTRACT}

BACKGROUND. Two sequelae of non-alcoholic steatohepatitis (NASH), ESLD and HCC, have become the leading causes for liver transplantation in the Western. The present study aims to approach the cellular metabolic disturbances involved in NASH progression that are associated with microbiota community changes.

METHODS. Metabolic effects and microbiota community changes were explored in the murine with NASH progression by blocking the Na/K-ATPase/Src/reactive oxygen amplification loop using the synthetic targeting peptide pNaKtide. DNA from the terminal ileum microbiota habitat was obtained and amplified by PCR to develop DNA bacterial phylogenic sequence analysis of wild type and treated animals at 12, 24 and 48 weeks. Induced changes by pSrc normalization at 24 weeks were correlated with liver morphological changes, intestinal $\mathrm{CD} 4^{+} / \mathrm{CD} 8^{+}$ratio, and liver macrophage CD14 ${ }^{+}$expression. Differences among groups were evaluated by ANOVA/ttest and Principal Component Analysis (PCA).

RESULTS. Microbiota communities varied significantly at all time points (12, 24 and 48 weeks), with an increase of Verrucomicrobia and a decrease of Bacteroidetes and Firmicutes in the HFD group. Microbiota community changes regressed to their wild-type state at 24 weeks on treated animals, and those changes were associated with a decrease in liver inflammation and senescence, lower ileum CD4 ${ }^{+} / C D 8^{+} T$ cells and higher liver CD14 ${ }^{+}$cells $(p<0.05)$.

Concomitantly, the metabolic disturbances in our diet-induced NASH model were normalized by NKA/Src signaling blockage and exercise with a paucity of apoptotic activity, mitigation of cell senescence, and regression of liver fibrosis $(p<0.01)$.

CONCLUSIONS. pSrc inhibition at caveolar $\alpha 1-\mathrm{Na} / \mathrm{K}-\mathrm{ATP}$ ase rescinded NASH-related metabolic disturbances establishing resident physiological microbiota communities with concomitant paucity on apoptotic activity and regression of liver fibrosis; effects that were associated with both gut and liver T-lymphocyte responses.

Word count: 276 
Schade \& Sanabria et al. NKA/Src in NASH progression

\section{INTRODUCTION}

Non-alcoholic Fatty Liver Disease (NAFLD) is a metabolic disease on the rise primarily due to the obesity epidemic affecting 2 billion of the world's population. ${ }^{1}$ NAFLD affects $20-25 \%$ of the US and its progression to non-alcoholic steatohepatitis (NASH) and related cirrhosis and HCC is presently the highest growing cause for liver transplantation, outpacing other forms of chronic liver disease, such as HBV, HCV, and alcoholic liver disease. ${ }^{2,34}$ In addition, HCC is concurrently becoming the most rapid growing cause of cancer-related mortality in the world, ${ }^{5}$ which occurs more often in non-cirrhotic livers in the background of NASH. ${ }^{6-8}$ The metabolic syndrome (obesity, HTN, and diabetes) is the most significant risk factor for NASH; $36.1 \%$ of adult men and $32.4 \%$ of adult women had metabolic syndrome in 2010 , at an estimated medical cost as high as $\$ 209.7$ billion. ${ }^{9,10}$ A fundamental cellular disarrangement present in over $80 \%$ of cells in chronic liver disease is cell cycle arrest, and its evolution to apoptosis is linked to disease progression and HCC. ${ }^{6,11}$ Cell senescence has been associated with increased production of reactive oxygen intermediates (ROI) from mitochondrial dysfunction, and telomerase shortening. ${ }^{12-14,15}$ Additionally, insulin resistance is associated with the induction and progression of cellular senescence and metabolic disarray. ${ }^{6}$ Disturbances in both glutathione sp. and metabolic prints have been documented in both animal models and patients with chronic liver disease and $\mathrm{HCC},{ }^{16-20}$ and authors have hypothesized the Gut-Liver axis as a key modulator in NASH progression, where metabolites and endotoxins produced in the gut are transported through the portal vein, enhancing metabolic disturbances and the inflammatory response in the liver. ${ }^{21}$ Gut microbiota communities have been shown to have a large role in not only NAFLD progression to NASH, but also in the cellular senescent state tied to HCC development. ${ }^{22}$

The ion-motive Na/K-ATPase (NKA), discovered as the first member of the P-type ATPase family, is also a membrane receptor that assembles with signaling partners to regulate fundamental cellular processes such as growth and division. ${ }^{23-29} \mathrm{SrC}$ - and ROI- dependent signaling through NKA $\alpha 1$, the only $\alpha$ isoform of NKA expressed in the liver, is the backbone of the best described branch of NKA signaling to date. A dysregulation of this pathway upon cellular redox unbalance has become abundantly evident in several metabolic and cardiovascular diseases, in which the NKA/Src/ROI loop self-perpetuates and over-amplifies $\mathrm{ROI}$ production. ${ }^{26,30}$ Most relevant in the context of the present study, we have observed that prophylactic blockade of NKA/Src/ROI amplification with pNaKtide, a 33-amino acid long peptide derived from a1-NKA sequence, ${ }^{31-33}$ attenuates the development of steatohepatitis in the C57BL/6J mouse on a Western diet. ${ }^{34,35} \mathrm{In}$ this model, administration of pNaKtide reduced obesity, minimized hepatic steatosis, inflammation and fibrosis, and markedly improved mitochondrial fatty acid oxidation, insulin sensitivity, and dyslipidemia. Therefore, NKA/Src/ROI could be a novel therapeutic target in NASH progression, with pNaKtide as a potential first-inclass synthetic candidate compound. This prospect prompted us to conduct a longitudinal efficacy study in a preclinical model. Specifically, we aimed to determine, in a mouse model that offers a close resemblance to the metabolic and morphological features observed clinically variations on the microbiota communities and T-cell response, and correlate such variations with the efficacy of pNaKtide vs. exercise on hepatic cellular disarrangements from established $\mathrm{NASH}$. To circumvent metabolic confounding factors due to well established propensity of male mice to develop malignancy in the NASH model, ${ }^{36}$ females were used exclusively in the present study. While a protocol was designed to document hepatic oxi-redox status, apoptotic activity, and parenchymal inflammation every 4 weeks, in a side-by-side comparison of pNaKtide vs. exercise, alternative protocol aimed to observed microbiota evolution under high fat diet for up to 48 weeks. 
Schade \& Sanabria et al. NKA/Src in NASH progression

\section{METHODS \\ Animal Model \& Experimental Design}

Seven-week-old female C57BL/6J mice from Jackson Laboratory (Farmington, CT) were housed following a $12 \mathrm{~h}: 12 \mathrm{~h}$ light-dark cycle under a temperature and humidity-controlled environment. Following acclimation, mice were fed ad libitum with a standard mouse chow (NMC, Bio-Serv, NJ) or a Western diet consisting of a high-fat diet (HFD, Bio-Serv, NJ, $60 \%$ of calories from fat) complemented with $55 \%$ fructose-in-water for 12,24 , or 48 weeks $(n=8$ per group to compensate for animal drop off). Authors have shown that mice exposed to a HFD for 12 weeks develop fatty livers with NASH and progressive fibrosis. ${ }^{37-40}$ After 12 weeks, a subset of mice were randomized into control and treatment groups and the study continued for an additional 12 weeks as follows: 1) NMC group, 2) HFD group, 3) HFD saline group, HFD plus normal saline (100 $\mu \mathrm{l} 0.9 \% \mathrm{NS}$, IP once a week), 4) pNaK group, HFD plus pNaKtide (25 mg/kg TBW dissolved in $100 \mu \mathrm{l} 0.9 \%$ NS, IP once a week), and 5) Exercise group, HFD plus exercise (30 minutes standardized protocol on an endless rotating wheel motivated by electroshock, five times/week). The experimental chart flow is shown in the supplemental material (Figure A1). Animal care underwent under ethical approval, and animal procedures followed guidelines from the University Institutional Animal Care and Use Committee (IACUC).

\section{Blood and tissue collection}

At week 12, 16, 20, 24, and 48 a sub cohort of mice from each study group was sedated with pentobarbital ( $5 \mathrm{mg} / \mathrm{kg}$ TBW, IP). At laparotomy and under magnification, blood was drawn from the infra-hepatic vena cava (IVC), followed by liver excision. Livers were washed with $0.9 \% \mathrm{NS}$ at room temperature and divided before being snap-frozen in liquid nitrogen and stored at $-80^{\circ} \mathrm{C}$ for later use or fixed at $4^{\circ} \mathrm{C}(10 \%$ formaldehyde $)$.

Concomitantly, terminal $10 \mathrm{cms}$ ileal samples were collected to have genomic DNA extracted from ileal contents, and DNA concentrations were measured on a Qubit fluorometer (ThermoFisher Scientific; Carlsbad, CA). lleum contents were collected by opening the ileum tube, exposing its surface, and scraping out the contents with a sterile plastic inoculation loop. Microbes were identified by sequencing of the $16 \mathrm{~S} v 3-\mathrm{v} 4$ hypervariable region. Illumina compatible libraries were constructed using a two-steps PCR amplification of the v3-v4 region with the addition of combinatorial dual-indices based on method described in Fadrosh et al. ${ }^{28}$ High Sensitivity Bioanalyzer Chips (Agilent, Santa Clara, CA) were used to assess amplicon size and quality. Amplicon libraries were pooled and sequenced in the Rapid Run mode on an Illumina HiSeq1500 instrument in a 2 X 270 paired end run with standard Illumina sequencing protocols at the University Genomics Core Facility. Phylogenetic analysis of $16 \mathrm{~S}$ data was performed by sequencing reads imported into QIIME2 version 2018.11, and sample sequences were inferred using the DADA2 plugin. Phylogenetic trees were constructed using mafft plugin. Alpha diversity was computed using Faith's Phylogenetic Diversity. Data were filtered into groups for individual comparisons, which were performed using Analysis of Composition of Microbiomes. All phylogenetic analyses and visualizations were performed in QIIME2.

\section{Immunohistochemistry}

Gut $C D 4^{+} / C D 8^{+}$. A set of ileum tissue slides were stained using immunohistochemistry (IHC) techniques with a FLEX monoclonal mouse anti-human CD4 antibody (Clone 4b12, Code IS649, from DAKO, Denmark) and a FLEX monoclonal mouse anti-human CD8 antibody (Clone C8/144B, Code IS623 from DAKO, Denmark). 40x images were then taken using a Zeiss brightfield microscope and analyzed using ImageJ 2.0. Scoring was determined as the number of positively stained lymphocytes/total number of cells per field. 
Schade \& Sanabria et al. NKA/Src in NASH progression

Liver CD14+. Liver tissue slides were stained using a monoclonal mouse anti-CD14 antibody ([4B4F12], ab182032 from Abcam, Chicago, IL) and counterstained with Hematoxylin QS. Images were taken at 40x with a Zeiss brightfield microscope and analyzed using ImageJ 2.0. Scoring was determined as the number of positively stained cells/number of cells per field.

\section{Metabolic Body Composition}

Metabolic body composition was assessed by EchoMRI-100H Body Composition Analyzer (Houston, TX). Records of total body weight (TBW), body water content, lean body mass, and body fat mass were recorded using GraphPad V7.04 (Loyola, CA, licensed to the University).

\section{Metabolomics: Plasma Treatment and Mass Spectrometry}

Materials and reagents, treatment of the plasma and specific liquid-chromatography-mass spectrometry methods have been described previously to measure glutathione sp. and nontarget metabolites ( $n=81$ metabolites, detailed description in supplemental materials). 16,18,19,41

\section{Gene Expression, Protein Expression and Activity}

The effects of diet \pm interventions on lipid metabolism and insulin sensitivity were assessed by the expression of the peroxisome proliferator-activated receptor (PPARY), its transcriptional coactivator PGC1a (RT-PCR), and FoxO1 \& Src expressions (Western blotting). The Hepatic $\mathrm{Na} / \mathrm{K}-\mathrm{ATPase}$ activity was determined as previously described. ${ }^{42}$ Details of the RT-PCR and pump activity method including a list and source of antibodies used can be found in the supplemental material.

\section{Morphological Assessment}

Liver NAFLD Activity Score (NAS) was determined by grading five images at 40x magnification on liver slides H\&E stained. Grading was carried out in accordance with standard grading criteria for macro-vesicular steatosis, micro-vesicular steatosis, inflammatory cell infiltrate and cellular hypertrophy (Figure A2 and A3A). ${ }^{43-47}$ Liver fibrosis was determined by grading five images at 40x magnification of Trichrome stained liver slides on a categorical scale by the fibrosis score (Figure A3B). ${ }^{48-50}$ Liver apoptotic activity was determined through grading five images at 40x magnification of terminal deoxynucleotidyl transferase (TdT)-mediated dUTP nick end labeling (TUNEL) stained liver slides (Figure A3C, Click-iT Plus TUNEL assay kit, Invitrogen by Thermo Fisher Scientific, MA). ${ }^{51-56}$ Lastly, liver cellular senescence was determined by grading five images at 40x magnification of liver slides stained for $\beta$-Galactosidase activity (Figure A3D, Cell signaling Technology \#9860, MA). Digitally recorded images at 40x magnification on the label (but blinded liver slides), were saved using ImageJ1.51u software. See supplemental material for details.

\section{Statistical Analysis}

Parametric data were examined with analysis of variance (ANOVA). Individual group means were compared by t-test employing the Holm-Sidak correction for multiple comparisons. Nonparametric data were analyzed with the Kruskal-Wallis test and individual groups compared by the chi-square $\left(\mathrm{X}^{2}\right)$ test. Principal component analysis (PCA) was conducted to investigate and visualize the pattern of metabolite differences among groups including log transformation for the construction of heat maps (Figure A4, in supplemental material). All analyses were carried out using the R-language and environment, a platform from the R project for statistical modeling, computing, and graphics (http://www.r-project.org/). See supplemental material for further details. 
Schade \& Sanabria et al. NKA/Src in NASH progression

\section{RESULTS}

\section{Microbiota and Gut-Liver Environment}

Microbiota Community Variations at 12, 24, and 48 Weeks. The proportion of Bacteroidetes was consistently decreased in the HFD vs. the NMC group at 12, 24 and 48 weeks (Figure 1A). In contrast, a greater proportion of Verrucomicrobia was found in the HFD group at 24 and 48 weeks. The proportion of Firmicutes varied, with a significantly higher proportion at 12 weeks, and a switch to a lower proportion at 24 and 48 weeks in the HFD vs. NMC. Various other levels of microbiota exhibited minor changes (less than 10\%). Marginal increases in Actinobacteria were observed at 12 and 24 weeks, and in Proteobacteria at 24 weeks in the HFD group.

Gut Microbial Community of experimental groups at 24 weeks. The proportion of Bacteroidetes and Firmicutes was decreased and the proportion of Verrucomicrobia was increased in the HFD group when compared to NMC, following similar changes as described above. Most notably, the pNaK group demonstrated a microbial profile almost identical to NMC (Figure 3B). The microbial profile of the Exercise group was similar to the HFD group, displaying an excess in Verrucomicrobia. Additionally, the groups of HFD, pNaK, and exercise all demonstrated slightly raised levels of Actinobacteria compared to NMC.

Gut's T-Cell \& Liver CD14+ Cell variations 24 Weeks. A significant decrease in $\mathrm{CD}^{+}$cells was observed in the pNaK treated group when compared to NMC, while a significant decrease in $\mathrm{CD}^{+}$cells in pNaKtide and Exercise groups was observed compared to NMC (Figure $2 \mathrm{~A}$, $\mathrm{p}<0.05$, t-test). The combined population of $\mathrm{CD} 4^{+} \& C D 8^{+}$cells followed a similar trend of being significantly decreased in pNaKtide and Exercise groups compared to NMC. There was no significant difference in $\mathrm{CD} 4^{+} / \mathrm{CD} 8^{+}$ratio among groups. In contrast, liver $\mathrm{CD} 14^{+}$cells were significantly increased in the pNaKtide and Exercise groups compared to NMC (Figure 2B, $\mathrm{p}<0.05$, t-test).

Metabolomics. To further evaluate the metabolic impact of $\mathrm{pNaK}$ and exercise, non-target metabolomic prints in plasma were performed. The metabolomic prints from the HFD group differed significantly from the NMC group, and the pNaKtide and exercise groups $(p<0.05$, by PCA). Metabolite prints separated experimental groups by diet HFD vs. NMC and by intervention HFD with NO intervention vs. HFD With intervention (Figure 3A, p<0.01). Metabolite data was Log ${ }^{10}$ transformed, and R-lab software was directed to display the data as visual arrays of NMC vs. HFD, NMC vs. pNaKtide, and exercise vs. NMC (Figure 3B). The displayed metabolic heat print from NMC vs. HFD was significantly different from the metabolic print where the NMC group was compared to the pNaKtide or exercise groups at 24 weeks $(p<0.01, P C A)$. Due to the log transformation, differences between groups are by a factor of 1:100 to 1:1000.

The aims of the present study did not include a detailed description of the metabolic pathways noted in NASH. Nevertheless, additional information is provided for metabolites concentrations. They were categorized into amino-peptides, carbohydrates, and lipids (Table 1, Supplemental material). Some compounds differed among groups by diet (NMC vs. HFD), by the intervention (HFD no intervention vs. HFD with intervention), or by both diet and intervention. Each metabolite was listed alphabetically, and its concentration was displayed as a box plot among groups with its statistically significant variation (accessible at https://public.tableau.com/ profile/joseph.shapiro\#!/vizhome/Juan metabolomics v3/Dashboard1?publish=yes). Differences in amino acids were noted, among others on coumaric, hippuric and kynurenic acids, thymidines, uridines, and zeatins $(p<0.05)$. Most sugars with significant variation were substrates involved in the Kreb's cycle. Adipic acid, arachidonic acid, corticosterone, myristic, oleic, and palmitic acids were among other lipids that significantly changed in the HFD groups when compared to other groups. Our group has described that glutathione sp., lactate, pyruvate, and glycerol were able to discriminate subjects with normal liver vs. subjects with 
Schade \& Sanabria et al. NKA/Src in NASH progression

ESLD. ${ }^{19}$ In the present study the production of lactate, pyruvate, and glycerol was undetectable in most animals during NASH progression. Nonetheless, changes in glutathione sp., butyrate and glucose were significant. The effects of pNaKtide and exercise were observed on aminoacids, carbohydrates, and lipids metabolism when compared to HFD.

\section{Effects of pSrc Normalization and Exercise on HFD-induced NASH}

The timing of intervention in mice subjected to this longitudinal efficacy study of pNaKtide vs. exercise in NASH is shown in supplemental Figure A1. The effect of the diet regimen was first characterized by a significant and time-dependent increase of total body weight (TBW) that occurred in the HFD groups compared to the NMC group from week 12 to week 24 (Figure 4A, $\mathrm{p}<0.05$, ANOVA). Echo-MRI analysis further revealed that the gain of TBW induced by the diet was due to an expansion of the fat mass compartment. Metabolic weight composition and plasma variations correlated with hepatic morphological changes manifested by a significant increase of macro- and micro-vesicular deposition of fat within parenchymal cells (ballooning) and larger inflammatory nests, when compared to the NMC group at week 24 (Figure 4B, NAS Score, $\mathrm{p}<0.05$, by $\mathrm{x}^{2}$ ). Additionally, there was a significant increase in fibrosis, apoptosis, and senescence activity in the HFD groups compared to the NMC group at week 24 (fibrosis score, apoptotic and senescence activity in $\%, p<0.05$ by $x^{2}$ ). Plasma glucose concentration, used as a surrogate of insulin resistance, was significantly higher in the HFD groups when compared to the NMC group at week 24 (Figure 4C, p<0.05, ANOVA). Concomitantly, glutathione reduced $(\mathrm{GSH})$ and glutathione oxidized (GS:SG) in plasma showed significant disturbances in the HFD groups compared to the NMC group at week 24 ( $p<0.05$, ANOVA). No significant difference was noted between HFD groups, excluding any effect of the vehicle or the administration method (IP).

After 12 weeks of HFD, randomization was performed with assignment of animals to no intervention (HFD), pNaKtide injections, or exercise. No difference in TBW or fat mass was detected among animals subjected to HFD for an additional 12 weeks, with or without pNaKtide or exercise (Figure 5A, Week 24, p>0.05, ANOVA). A dramatic difference was observed in apoptotic and cell senescence activities among groups by week 24 (Figure 5B). Specifically, there was a significant decrease in cell senescence activity, by week 16 with the abrogation of apoptosis by week 24 in both intervention groups (pNaK and exercise), when compared to the HFD group $\left(p<0.01\right.$, by $\left.x^{2}\right)$. Cell senescence was significantly increase in the exercise group compared to the HD or pNaKtide groups by week $24(p<0.05)$. Concomitantly, there was a significant difference in the fibrosis score among groups, with progression to fibrosis noted in all HFD groups, but with a clear attenuation of the fibrotic pattern in the intervention groups by week $24\left(p<0.01\right.$, by $\left.x^{2}\right)$. There was a significant difference in the NAS score of the intervention groups compared to the groups at week $24\left(p<0.05\right.$, by $\left.x^{2}\right)$. Each component of the NAS score (macro-vesicular steatosis, micro-vesicular steatosis, cell hypertrophy, and inflammatory foci) was evaluated separately (Figure A2, supplemental material). The inflammatory component was more significant in the HFD groups with its peak at week 24 when compared to the NMC and intervention groups $\left(p<0.05\right.$, by $\left.x^{2}\right)$. Similar levels of inflammation were observed between the NMC and intervention groups by week 24 ( $p>0.05)$. Glucose concentration was robustly and significantly decreased in both pNaKtide, and exercise groups compared to the untreated HFD group at week 24 (Figure $5 C, p<0.05$ by ANOVA). In addition, there was a significant difference in the glutathione sp. (glutathione reduced ${ }^{\mathrm{GSH}}$, glutathione oxidized ${ }^{\mathrm{GS}} \mathrm{SG}$ ) among groups $(p<0.05$, ANOVA). Specifically, the pNaKtide and exercise groups had significantly lower concentrations of GSH and GS:SG in their plasma than the untreated HFD group at week 24.

\section{Expression and Activity of Key Hepatic Regulators of Metabolic Signaling}

The expression of the peroxisome proliferator-activated receptor-y (PPARY), and its transcriptional coactivator PGC1 $\alpha$ was significantly increased in the pNaKtide group when 
Schade \& Sanabria et al. NKA/Src in NASH progression

compared to the HFD group (Figure $5 A \& B$, respectively, $p<0.05$, by ANOVA). The transcription of PPARy peaked in the $\mathrm{pNaK}$ and exercise groups at week 16 to return to similar levels when compared to the other groups by week 20 . Similar behavior was observed for the expression of PGC1a. Nevertheless, a second and significant peak was observed in the exercise group at week 24 when compared to the pNaKtide group. While FoxO1 expression was significantly upregulated in the pNaKtide and exercise groups, Src phosphorylation peaked in the HFD group at week 20 (Figure 5 C\&D). Liver Src kinase expression and activation as the ratio Srcphosphorylated over total Src (pSrc/pSrc+CSrc) was significantly different among groups $(p<0.05$, by ANOVA). Specifically, there was a paucity of pSrc in the pNaKtide and exercise groups.

\section{Hepatic Na/K-ATPase activity and $\alpha 1$ Isoform expression}

A significant difference in total hepatic $\mathrm{Na} / \mathrm{K}-\mathrm{ATP}$ ase activity was detected at week 24 among groups (Figure $5 E$ ). The maximal ouabain-inhibitable ATPase activity was significantly higher in the livers of mice in the pNaKtide group compared to the HFD or exercise groups $(p<0.05$, ANOVA followed by t-test). This was not associated with a significant difference in the total expression of $\mathrm{Na} / \mathrm{K}-\mathrm{ATP}$ ase $\alpha 1$ subunit among the groups at week 24 . 
Schade \& Sanabria et al. NKA/Src in NASH progression

\section{DISCUSSION}

The global incidence and prevalence of chronic liver disease and its sequelae ESLD and HCC are increasing as a consequence of both viral hepatitis ( $B$ and $C$ ) and the continuous spread of the metabolic disturbances related to the obesity epidemic. ${ }^{57,58} \mathrm{NASH}$ and NAFLD development are characterized by a series of metabolic disturbances resulting from an increase in body fat, glucose intolerance, and an accumulation of fatty acids. These disturbances are known together as the metabolic syndrome, manifested in the liver as NAFLD, and its progression to NASH is further marked by increased oxidative stress, fibrosis, and apoptosis. ${ }^{59,60}$ The two-hit theory, where the increased fat diet and insulin resistance leave the liver vulnerable to a second hit from oxidative stress, leads cells through an increased inflammatory storm that results in apoptosis and progression to fibrosis. ${ }^{60}$ Our HFD induced NASH rodent model emulated the liver histological changes clinically observed from known dysfunctional mitochondrial $\beta$-lipid oxidation with further insulin resistance status and progressive exhaustion of the cell oxi-redox reserves. The novelty of the present study resides in the link between the gut microbiota community variations that occur during a HFD regimen with NASH progression, and its regression by pSrc normalization at the a1-NKA associated with immunological changes at the liver-gut axis. A decrease in the proportion of Bacteroidetes (anaerobes) with a concomitant increase in Verrucomicrobia are associated with NASH inflammatory milieu progression, enhanced cellular senescence, increased apoptotic activity and liver fibrosis. Interestingly, blockage of Src-phosphorylation at the cell membrane by pNaKtide, restored physiological microbiota communities, which were associated with wild type liver morphology from rescinded apoptotic activity and collagen deposition. Concomitantly, ileum CD4 ${ }^{+}$and $\mathrm{CD}^{+} \mathrm{T}$-cells decreased, remaining liver CD14 ${ }^{+}$highly expressed. The molecular mechanism of protection by pNaKtide, differed from the one induced by exercise, restoring $\mathrm{Na} / \mathrm{K}-\mathrm{ATP}$ ase activity without changes in the NKA a1 subunit expression.

Firmicutes, Bacteroidetes, Actinobacteria, Proteobacteria, and Verrucomicrobia are considered the largest contributors to the microbial gut community. ${ }^{61,62}$ Furthermore, Bacteroidetes are gram negative and Firmicutes are gram positive bacteria, with both ranging from anaerobic to aerobic strands, making up for approximately $90 \%$ of the microbiota community. ${ }^{62}$ In the longitudinal study of the microbiota over 48 weeks, the three greatest changes were observed in Bacteroidetes, Firmicutes, and Verrucomicrobia. The ratio of these bacteria has been studied to act as a correlational marker in both obesity and GI health. ${ }^{63,64}$ However, it should be noted that reports in gut microbiota community alterations have not been consistent. ${ }^{62,65}$ For instance, in one study Firmicutes exhibited a greater effect in establishing NAFLD compared to Bacteroidetes, while another study demonstrated that lowering both Firmicutes and Bacteroidetes led to improvement in diet-induced obese mice. ${ }^{66,67}$ Although no consensus has been reached between microbiota changes and NAFLD/NASH development, its association with NASH progression has been consistently observed. ${ }^{68}$ In the present study, Verrucomicrobia was higher at 12, 24, and 48 weeks in the HFD group compared to NMC. Furthermore, Bacteroidetes was decreased at 12, 24, and 48 weeks, albeit to a lesser degree at 24 weeks in the HFD group compared to NMC. Firmicutes levels were initially higher at 12 weeks; evolving to lower frequency at 24 and 48 weeks in HFD compared to NMC. Furthermore, a marginal increase in Actinobacteria at 12 and 24 weeks occurred in the HFD group compared to NMC, as well as Proteobacteria at week 24. Actinobacteria is a gram positive, anaerobic bacteria, while proteobacteria is a gram negative bacteria that has been observed to have a positive correlation with fat intake. ${ }^{69}$ Changes of gut microbiota at 24 weeks were further investigated under treatment with pNaKtide and exercise. At 24 weeks, pSrc normalization restored gut microbiota community to resemble NMC, changes that were not observed in the exercise group, which resembled a HFD microbiota profile. 
Schade \& Sanabria et al. NKA/Src in NASH progression

One would expect the immune system to mirror changes in microbiota, due to the relationship between the gut microbiota community and the gut intestinal immune system. The microbiota profile under pNaKtide treatment showed a similar microbiota community to NMC at 24 weeks, yet the ileal number of $\mathrm{CD}^{+} \& \mathrm{CD}^{+}$significantly differs from the NMC group. One possible explanation for this discrepancy is that Src-phosphorylation does not influence microbiota community changes through an immune response, but through metabolic alterations that exercise not only locally, but through a gut-liver axis effect. A decreased response from the immune system is often observed in individuals with NAFLD, suggesting that the gut microbiota and immune system have developed a coevolutionary relationship. ${ }^{70}$ Patients with NAFLD had lower duodenal $\mathrm{CD}^{+} \& \mathrm{CD} 8^{+}$counts when compared to healthy patients, ${ }^{70}$ which correlate with increased intestinal inflammation manifested by higher TNF- $\alpha / \mathrm{lL}-6$ mRNA expression.

Furthermore, pre-clinical and clinical studies in NASH related HCC have shown lower counts of $\mathrm{CD}^{+} .{ }^{71}$ Specifically, the enrichment of linoleic acid in the cirrhotic microenvironment of NASH patients promotes disruption of mitochondrial function in a greater proportion than other fatty acids, such as palmitic acid. As $\mathrm{CD}^{+} \mathrm{T}$ lymphocytes have a larger mitochondrial load than $\mathrm{CD}^{+}$T-lymphocytes, they not only generate more mitochondrial derived ROS, but CD4 ${ }^{+}$cells may undergo larger selective loss of mitochondrial function and viability. Therefore, disruption of mitochondrial function by linoleic acid mediates selective loss of intrahepatic CD4 ${ }^{+} \mathrm{T}$ -

lymphocytes, a state often associated with HCC. Another possible explanation for lowered ileal $\mathrm{CD}^{+}$and $\mathrm{CD}^{+}$lymphocytes is the heightened levels of lipopolysaccharides observed in NASH dysbiosis. Lipopolysaccharides originates from gram-negative bacteria wall, and several studies have shown that lipopolysaccharide variations have an apoptotic effect on CD4 ${ }^{+}$and $\mathrm{CD} 8^{+} \mathrm{T}$ cells. ${ }^{72,73}$ Specifically, the receptor target for lipopolysaccharides is Toll-Like Receptor 4, which resides primarily on Kupfer cells in the liver. ${ }^{74}$ Lipopolysaccharides produced in the gut travel through the portal vein to the liver, where they bind to receptors on Kupfer cells and activate a pro-inflammatory response. ${ }^{75}$ Various studies have implicated an association between lipopolysaccharides percolation rates and CD14 in pathophysiology of fibrosis, insulin resistance, and apoptotic activity observed in $\mathrm{NASH} .{ }^{76-78}$ In addition to the membrane bound CD14 found on Kupfer cells (mCD14), a soluble CD14 (sCD14) is present and produced by hepatocytes. ${ }^{79} 80,81$ Although we did not measure sCD14 in the present study, CD14+ activation was thereby determined by positively identified Kupfer and hepatocyte $\mathrm{mCD} 14^{+}$expression, where a significant increase was observed in both the pNaKtide, and exercise groups compared to NMC at 24 weeks.

Metabolomic signatures were different in the HFD, pNaKtide, and exercise groups when compared to the NMC group. The visual display of metabolic heat maps could potentially serve as a surrogate for the diagnosis of liver disease in a patient, for their progression or response to treatment, and the early detection of the development of ESLD and HCC. Indeed, metabolic prints were able to discriminate patients with healthy livers from patients at different stages of ESLD, as judged by the MELD score. ${ }^{19}$ Furthermore, metabolic prints may help to discriminate patients with ESLD by tumor status. ${ }^{19,82} \mathrm{OH}$-butyrate was found to be an early biomarker of insulin resistance and glucose intolerance in non-diabetic subjects. ${ }^{83}$ pNaKtide normalized the liver's oxi-redox status by decreasing ROI through a circuit that restored physiological mitochondrial $\beta$-lipid oxidation and insulin sensitivity; pathways included the upregulation of both PPARy-PGC1a complex and FOXO1. Both proteins enhance the effects of insulin, and the PPARy-PGC1a complex is involved in mitochondrial $\beta$-lipid oxidation. Increased permeability of mitochondrial membrane pores amplified the leakage of intermembrane cytochromes and activation of caspase processes, which may explain the morphological peak of apoptosis at week $24 .{ }^{84,85}$ The apoptotic activity has been correlated with the progression of liver disease to an end-organ stage ${ }^{55,86}$ Exercise may drive metabolic changes through a sarcoplasmic-energy burning mechanism associated with the mobilization of lipid droplets from the liver. This line of 
Schade \& Sanabria et al. NKA/Src in NASH progression

thought may explain, at least in part, the increasing concentration of fatty acids (i.e., arachidonic, linoleic, palmitic acids) in liver cells in the pNaKtide group when compared to the HFD and exercise groups (Table 1, supplemental material).

We validated our HFD induced NASH murine model by reproducing the described clinical observations of increase in body fat, altered liver morphology, and known metabolic disturbances in $\beta$-lipid oxidation, insulin resistance and glutathione sp. Glutathione reduced, and glutathione oxidized significantly increased in the HFD groups when compared to the other groups, more so at weeks 20 and 24, when peaks in cell arrest and apoptosis were noted. Although the $\mathrm{HO} 1$ expression was significantly higher in the HFD groups when compared to the NMC group at week 24, similar expression of $\mathrm{HO} 1$ was observed in all HFD groups (Figure A5, supplemental material). Furthermore, we were able to reproduce the previously described changes by our group on NASH prevention and progression. $33,87,88$ The proportion of aging cells expressing the senescence phenotype was progressively increased from weeks 12 to 16 in the HFD groups, with a dramatic increase in the apoptotic activity at week 24 when compared to the NMC group. pNaKtide and exercise were able to prevent the peak on apoptosis observed at week 24 with a progressive decrease in the proportion of cells expressing the senescent phenotype, likely in the HFD group from cells going to apoptosis, and in the pNaKtide group from reversing cell senescence, a change associated with a decrease in the inflammatory score. Furthermore, protein function may be affected by increased ROI through protein carbonylation. ${ }^{34,89}$ Normalization of the Src kinase pool bound to the $\alpha 1-N K A$ by pNaKtide brought to physiological levels both hydrolytic and signaling a1-NKA protein functions.

The results of the present studies should be perceived under their limitations. The HFD-induced NASH model in the rodent resembles, to an extent, the clinical manifestations of NASH and the metabolic syndrome observed in humans. Nonetheless, the response to interventions and its pathways may differ significantly. Gut microbial community changes have been rarely reported consistently and must be carefully attributed to the specific experimental parameters of the current study. Furthermore, gut microbiota and intestinal immune system changes of all treatment groups were observed at 24 weeks, meaning a longitudinal correlation may exist that has not yet been identified. Although the present studies were controlled for animal variability by gender, age, circadian rhythm, and strict adherence to laboratory protocols, biological variability is unavoidable. The presence of metabolic syndrome has multiple organs as a target, and although plasma metabolites were associated with liver morphology and liver protein expression, the metabolic changes that occurred because of changes in other organs remain to be determined. The present study was performed under a relatively short period ( 24 weeks), and during this time interval, the development of an adverse event from the use of pNaKtide was not observed. Nevertheless, the study aimed to determine microbiota community variations and their association with the metabolic disturbances of $\mathrm{NASH}$, mitigating any effect due to the development of malignancy. In addition, the effects from normalizing the a1-Na/K-ATPase signalosome were assessed.

CONCLUSION. The described murine model of NASH develops fatty livers emulating the clinical liver changes of NASH associated with accelerated senescence/apoptotic cell activities induced by a decreased cellular oxi-redox status following metabolic signatures and changes in the microbiota communities. Normalization of the $\alpha 1-\mathrm{Na} / \mathrm{K}-\mathrm{ATP}$ ase signalosome rescinded metabolic changes with regression to organ wild-type morphology, restoring physiological microbiota communities. Furthermore, pSrc inhibition led to significantly lower gut $\mathrm{T}$ cells albeit conserving an increased and activated liver macrophages. 
Schade \& Sanabria et al. NKA/Src in NASH progression

\section{Acknowledgments}

The authors have no conflict of interest to declare and would like to thank the contributions of Dr. L. Dial from the Department of Medicine and Dr. S. Sigdel from the Department of Pathology at Marshall University, as well as Mr. C. Nypan, Mss. C. Taylor, Mr. G. Smith, Mss. J. Reuther, Ms. C. Nagisetty, and Ms. N Beltran for their commitment and help with the procedures of the present study. 
Schade \& Sanabria et al. NKA/Src in NASH progression

\section{REFERENCES}

1. Swinburn BA, Kraak VI, Allender S, et al. The Global Syndemic of Obesity, Undernutrition, and Climate Change: The Lancet Commission report. Lancet 2019;393(10173):791-846. DOI: 10.1016/S0140-6736(18)32822-8.

2. Bugianesi E, McCullough AJ, Marchesini G. Insulin resistance: a metabolic pathway to chronic liver disease. Hepatology 2005;42(5):987-1000. DOI: 10.1002/hep.20920.

3. Schwabe RF, Tabas I, Pajvani UB. Mechanisms of Fibrosis Development in Nonalcoholic Steatohepatitis. Gastroenterology 2020;158(7):1913-1928. DOI: 10.1053/j.gastro.2019.11.311.

4. Younossi Z, Stepanova M, Ong JP, et al. Nonalcoholic Steatohepatitis Is the Fastest Growing Cause of Hepatocellular Carcinoma in Liver Transplant Candidates. Clin Gastroenterol Hepatol 2019;17(4):748-755 e3. DOI: 10.1016/j.cgh.2018.05.057.

5. Hayes CN, Zhang P, Chayama K. The Role of Lipids in Hepatocellular Carcinoma. In: Tirnitz-Parker JEE, ed. Hepatocellular Carcinoma. Brisbane (AU)2019.

6. Dongiovanni P, Romeo S, Valenti L. Hepatocellular carcinoma in nonalcoholic fatty liver: role of environmental and genetic factors. World J Gastroenterol 2014;20(36):12945-55. DOI: 10.3748/wjg.v20.i36.12945.

7. Global Burden of Disease Cancer C, Fitzmaurice C, Akinyemiju TF, et al. Global, Regional, and National Cancer Incidence, Mortality, Years of Life Lost, Years Lived With Disability, and Disability-Adjusted Life-Years for 29 Cancer Groups, 1990 to 2016: A Systematic Analysis for the Global Burden of Disease Study. JAMA Oncol 2018;4(11):1553-1568. DOI: 10.1001/jamaoncol.2018.2706.

8. Karagozian R, Derdak Z, Baffy G. Obesity-associated mechanisms of hepatocarcinogenesis. Metabolism 2014;63(5):607-17. DOI: 10.1016/.metabol.2014.01.011.

9. Younossi Z, Anstee QM, Marietti M, et al. Global burden of NAFLD and NASH: trends, predictions, risk factors and prevention. Nat Rev Gastroenterol Hepatol 2018;15(1):1120. DOI: $10.1038 /$ nrgastro.2017.109.

10. Estes C, Razavi H, Loomba R, Younossi Z, Sanyal AJ. Modeling the epidemic of nonalcoholic fatty liver disease demonstrates an exponential increase in burden of disease. Hepatology 2018;67(1):123-133. DOI: 10.1002/hep.29466.

11. Aravinthan A, Shannon N, Heaney J, Hoare M, Marshall A, Alexander GJ. The senescent hepatocyte gene signature in chronic liver disease. Exp Gerontol 2014;60:3745. DOI: 10.1016/j.exger.2014.09.011.

12. Aini W, Miyagawa-Hayashino A, Ozeki M, et al. Accelerated telomere reduction and hepatocyte senescence in tolerated human liver allografts. Transpl Immunol 2014;31(2):55-9. DOI: 10.1016/j.trim.2014.06.008.

13. Aravinthan A, Mells G, Allison M, et al. Gene polymorphisms of cellular senescence marker p21 and disease progression in non-alcohol-related fatty liver disease. Cell Cycle 2014;13(9):1489-94. DOI: 10.4161/cc.28471.

14. Irvine KM, Skoien R, Bokil NJ, et al. Senescent human hepatocytes express a unique secretory phenotype and promote macrophage migration. World J Gastroenterol 2014;20(47):17851-62. DOI: 10.3748/wjg.v20.i47.17851.

15. Lade A, Noon LA, Friedman SL. Contributions of metabolic dysregulation and inflammation to nonalcoholic steatohepatitis, hepatic fibrosis, and cancer. Curr Opin Oncol 2014;26(1):100-7. DOI: 10.1097/CCO.00000000000000042.

16. Kombu RS, Zhang GF, Abbas R, et al. Dynamics of glutathione and ophthalmate traced with $2 \mathrm{H}$-enriched body water in rats and humans. Am J Physiol Endocrinol Metab 2009;297(1):E260-9. DOI: 10.1152/ajpendo.00080.2009. 
Schade \& Sanabria et al. NKA/Src in NASH progression

17. Abbas R, Kombu RS, Ibarra RA, Goyal KK, Brunengraber H, Sanabria JR. The dynamics of glutathione species and ophthalmate concentrations in plasma from the VX2 rabbit model of secondary liver tumors. HPB Surg 2011;2011:709052. DOI: 10.1155/2011/709052.

18. Andres Ibarra R, Abbas R, Kombu RS, et al. Disturbances in the glutathione/ophthalmate redox buffer system in the woodchuck model of hepatitis virusinduced hepatocellular carcinoma. HPB Surg 2011;2011:789323. DOI: 10.1155/2011/789323.

19. Sanabria JR, Kombu RS, Zhang GF, et al. Glutathione species and metabolomic prints in subjects with liver disease as biological markers for the detection of hepatocellular carcinoma. HPB (Oxford) 2016;18(12):979-990. DOI: 10.1016/j.hpb.2016.09.007.

20. Luo P, Yin P, Hua R, et al. A Large-Scale, Multicenter Serum Metabolite Biomarker Identification Study for hte Early Detection of Hepatocellular Carcinoma. Hepatology 2018;67(2):662-675.

21. Kirpich IA, Marsano LS, McClain CJ. Gut-liver axis, nutrition, and non-alcoholic fatty liver disease. Clin Biochem 2015;48(13-14):923-30. DOI: 10.1016/j.clinbiochem.2015.06.023.

22. Yoshimoto S, Loo TM, Atarashi K, et al. Obesity-induced gut microbial metabolite promotes liver cancer through senescence secretome. Nature 2013;499(7456):97-101. DOI: 10.1038/nature12347.

23. Banerjee M, Cui X, Li Z, et al. Na/K-ATPase Y260 Phosphorylation-mediated Src Regulation in Control of Aerobic Glycolysis and Tumor Growth. Sci Rep 2018;8(1):12322. DOI: 10.1038/s41598-018-29995-2.

24. Banerjee M, Duan Q, Xie Z. SH2 Ligand-Like Effects of Second Cytosolic Domain of $\mathrm{Na} / \mathrm{K}-\mathrm{ATP}$ ase alpha1 Subunit on Src Kinase. PLoS One 2015;10(11):e0142119. DOI: 10.1371/journal.pone.0142119.

25. Cai T, Wang $\mathrm{H}$, Chen $\mathrm{Y}$, et al. Regulation of caveolin-1 membrane trafficking by the $\mathrm{Na} / \mathrm{K}-\mathrm{ATPase}$. J Cell Biol 2008;182(6):1153-69. DOI: 10.1083/jcb.200712022.

26. Liu J, Tian J, Chaudhry M, et al. Attenuation of Na/K-ATPase Mediated Oxidant Amplification with pNaKtide Ameliorates Experimental Uremic Cardiomyopathy. Sci Rep 2016;6:34592. DOI: 10.1038/srep34592.

27. Xie J, Ye Q, Cui X, et al. Expression of rat Na-K-ATPase alpha2 enables ion pumping but not ouabain-induced signaling in alpha1-deficient porcine renal epithelial cells. Am J Physiol Cell Physiol 2015;309(6):C373-82. DOI: 10.1152/ajpcell.00103.2015.

28. Xie Z, Cai T. Na+-K+--ATPase-mediated signal transduction: from protein interaction to cellular function. Mol Interv 2003;3(3):157-68. DOI: 10.1124/mi.3.3.157.

29. Xie Z, Kometiani P, Liu J, Li J, Shapiro Jl, Askari A. Intracellular reactive oxygen species mediate the linkage of $\mathrm{Na}+/ \mathrm{K}+-\mathrm{ATP}$ ase to hypertrophy and its marker genes in cardiac myocytes. J Biol Chem 1999;274(27):19323-8. (https://www.ncbi.nlm.nih.gov/pubmed/10383443

http://www.jbc.org/content/274/27/19323.full.pdf).

30. Liu CC, Karimi Galougahi K, Weisbrod RM, et al. Oxidative inhibition of the vascular $\mathrm{Na}+-\mathrm{K}+$ pump via NADPH oxidase-dependent beta1-subunit glutathionylation: implications for angiotensin II-induced vascular dysfunction. Free Radic Biol Med 2013;65:563-572. DOI: 10.1016/j.freeradbiomed.2013.06.040.

31. Li Z, Zhang Z, Xie JX, et al. Na/K-ATPase mimetic pNaKtide peptide inhibits the growth of human cancer cells. J Biol Chem 2011;286(37):32394-403. DOI: 10.1074/jbc.M110.207597.

32. Sodhi K, Maxwell K, Yan Y, et al. pNaKtide inhibits Na/K-ATPase reactive oxygen species amplification and attenuates adipogenesis. Sci Adv 2015;1(9):e1500781. DOI: 10.1126/sciadv. 1500781. 
Schade \& Sanabria et al. NKA/Src in NASH progression

33. Sodhi K, Srikanthan K, Goguet-Rubio P, et al. pNaKtide Attenuates Steatohepatitis and Atherosclerosis by Blocking Na/K-ATPase/ROS Amplification in C57BI6 and ApoE Knockout Mice Fed a Western Diet. Sci Rep 2017;7(1):193. DOI: 10.1038/s41598-01700306-5.

34. Sodhi K, Nichols A, Mallick A, et al. The Na/K-ATPase Oxidant Amplification Loop Regulates Aging. Sci Rep 2018;8(1):9721. DOI: 10.1038/s41598-018-26768-9.

35. Sodhi K, Ouri N, Favero G, et al. Fructose mediated NOn-alcoholic Fatty Liver Disease is attenuated by HO-1-SIRT1 module in murine hepatocytes and mice fed a high fructose diet. PLOS one 2015;DOI: 10.1371.

36. Xie G, Wang X, Zhao A, et al. Sex-dependent effects on gut microbiota regulate hepatic carcinogenic outcomes. Sci Rep 2017;7:45232. DOI: 10.1038/srep45232.

37. Charlton M, Krishnan A, Viker K, et al. Fast food diet mouse: novel small animal model of NASH with ballooning, progressive fibrosis, and high physiological fidelity to the human condition. Am J Physiol Gastrointest Liver Physiol 2011;301(5):G825-34. DOI: 10.1152/ajpgi.00145.2011.

38. Hirsova P, Ibrahim SH, Bronk SF, Yagita H, Gores GJ. Vismodegib suppresses TRAILmediated liver injury in a mouse model of nonalcoholic steatohepatitis. PLoS One 2013;8(7):e70599. DOI: 10.1371/journal.pone.0070599.

39. Ibrahim SH, Hirsova P, Tomita K, et al. Mixed lineage kinase 3 mediates release of $\mathrm{C}-\mathrm{X}$ C motif ligand 10-bearing chemotactic extracellular vesicles from lipotoxic hepatocytes. Hepatology 2016;63(3):731-44. DOI: 10.1002/hep.28252.

40. Krishnan A, Abdullah TS, Mounajjed T, et al. A longitudinal study of whole body, tissue, and cellular physiology in a mouse model of fibrosing NASH with high fidelity to the human condition. Am J Physiol Gastrointest Liver Physiol 2017;312(6):G666-G680. DOI: 10.1152/ajpgi.00213.2016.

41. Ibarra R, Dazard JE, Sandlers Y, et al. Metabolomic Analysis of Liver Tissue from the VX2 Rabbit Model of Secondary Liver Tumors. HPB Surg 2014;2014:310372. DOI: $10.1155 / 2014 / 310372$.

42. Ismail-Beigi F, Edelman IS. The mechanism of the calorigenic action of thyroid hormone. Stimulation of Na plus $+\mathrm{K}$ plus-activated adenosinetriphosphatase activity. J Gen Physiol 1971;57(6):710-22. (https://www.ncbi.nlm.nih.gov/pubmed/4252666

http://jgp.rupress.org/content/jgp/57/6/710.full.pdf).

43. Abdel-Razik A, Mousa N, Shabana W, et al. A novel model using mean platelet volume and neutrophil to lymphocyte ratio as a marker of nonalcoholic steatohepatitis in NAFLD patients: multicentric study. Eur J Gastroenterol Hepatol 2016;28(1):e1-9. DOI: 10.1097/MEG.0000000000000486.

44. Ballestri S, Nascimbeni F, Romagnoli D, Lonardo A. The independent predictors of nonalcoholic steatohepatitis and its individual histological features.: Insulin resistance, serum uric acid, metabolic syndrome, alanine aminotransferase and serum total cholesterol are a clue to pathogenesis and candidate targets for treatment. Hepatol Res 2016;46(11):1074-1087. DOI: 10.1111/hepr.12656.

45. Cao Y, Pan Q, Cai W, et al. Modulation of Gut Microbiota by Berberine Improves Steatohepatitis in High-Fat Diet-Fed BALB/C Mice. Arch Iran Med 2016;19(3):197-203. DOI: 0161903/AIM.008.

46. Hannah WN, Jr., Harrison SA. Lifestyle and Dietary Interventions in the Management of Nonalcoholic Fatty Liver Disease. Dig Dis Sci 2016;61(5):1365-74. DOI: 10.1007/s10620-016-4153-y.

47. Linden MA, Sheldon RD, Meers GM, et al. Aerobic exercise training in the treatment of non-alcoholic fatty liver disease related fibrosis. J Physiol 2016;594(18):5271-84. DOI: 10.1113/JP272235. 
Schade \& Sanabria et al. NKA/Src in NASH progression

48. Bird B, Rowlette J. A protocol for rapid, label-free histochemical imaging of fibrotic liver. Analyst 2017;142(8):1179-1184. DOI: 10.1039/c6an02080a.

49. Cheung JS, Fan SJ, Gao DS, Chow AM, Man K, Wu EX. Diffusion tensor imaging of liver fibrosis in an experimental model. J Magn Reson Imaging 2010;32(5):1141-8. DOI: 10.1002/jmri.22367.

50. Kimm SY, Tarin TV, Monette S, et al. Nonthermal Ablation by Using Intravascular Oxygen Radical Generation with WST11: Dynamic Tissue Effects and Implications for Focal Therapy. Radiology 2016;281(1):109-18. DOI: 10.1148/radiol.2016141571.

51. Lee YS, Park JS, Lee DH, et al. The Antidiabetic Drug Lobeglitazone Protects Mice From Lipogenesis-Induced Liver Injury via Mechanistic Target of Rapamycin Complex 1 Inhibition. Front Endocrinol (Lausanne) 2018;9:539. DOI: 10.3389/fendo.2018.00539.

52. Kolachala VL, Palle SK, Shen M, Shenoi A, Shayakhmetov DM, Gupta NA. Influence of Fat on Differential Receptor Interacting Serine/Threonine Protein Kinase 1 Activity Leading to Apoptotic Cell Death in Murine Liver Ischemia Reperfusion Injury Through Caspase 8. Hepatol Commun 2019;3(7):925-942. DOI: 10.1002/hep4.1352.

53. Yin $\mathrm{H}$, Zhang $\mathrm{H}$, Kong $\mathrm{Y}$, et al. Apelin protects auditory cells from cisplatin-induced toxicity in vitro by inhibiting ROS and apoptosis. Neurosci Lett 2020;728:134948. DOI: 10.1016/j.neulet.2020.134948.

54. Farrell GC, Larter CZ, Hou JY, et al. Apoptosis in experimental NASH is associated with p53 activation and TRAIL receptor expression. J Gastroenterol Hepatology 2009;24(3):443-452.

55. Feldstein AE, Canbay A, Angulo $P$, et al. Hepatocyte apoptosis and fas expression are prominent features of human nonalcoholic steatohepatitis. Gastroenterology 2003;125(2):437-43. (https://www.ncbi.nlm.nih.gov/pubmed/12891546

56. Jiang $\mathrm{Y}$, Zhao M, An W. Increased hepatic apoptosis in high-fat diet-induced NASH in rats may be associated with downregulation of hepatic stimulator substance. J Mol Med (Berl) 2011;89(12):1207-17. DOI: 10.1007/s00109-011-0790-y.

57. Collaborators GBDO, Afshin A, Forouzanfar $\mathrm{MH}$, et al. Health Effects of Overweight and Obesity in 195 Countries over 25 Years. N Engl J Med 2017;377(1):13-27. DOI: 10.1056/NEJMoa1614362.

58. Stanaway JD, Flaxman AD, Naghavi M, et al. The global burden of viral hepatitis from 1990 to 2013: findings from the Global Burden of Disease Study 2013. Lancet 2016;388(10049):1081-8. DOI: 10.1016/S0140-6736(16)30579-7.

59. Koek GH, Liedorp PR, Bast A. The role of oxidative stress in non-alcoholic steatohepatitis. Clin Chim Acta 2011;412(15-16):1297-305. DOI: 10.1016/j.cca.2011.04.013.

60. Bessone F, Razori MV, Roma MG. Molecular pathways of nonalcoholic fatty liver disease development and progression. Cell Mol Life Sci 2019;76(1):99-128. DOI: 10.1007/s00018-018-2947-0.

61. Brandi G, De Lorenzo S, Candela M, et al. Microbiota, NASH, HCC and the potential role of probiotics. Carcinogenesis 2017;38(3):231-240. DOI: 10.1093/carcin/bgx007.

62. Liu Q, Liu S, Chen L, et al. Role and effective therapeutic target of gut microbiota in NAFLD/NASH. Exp Ther Med 2019;18(3):1935-1944. DOI: 10.3892/etm.2019.7781.

63. Stojanov S, Berlec A, Strukelj B. The Influence of Probiotics on the Firmicutes/Bacteroidetes Ratio in the Treatment of Obesity and Inflammatory Bowel disease. Microorganisms 2020;8(11). DOI: 10.3390/microorganisms8111715.

64. Fujio-Vejar S, Vasquez Y, Morales P, et al. The Gut Microbiota of Healthy Chilean Subjects Reveals a High Abundance of the Phylum Verrucomicrobia. Front Microbiol 2017;8:1221. DOI: 10.3389/fmicb.2017.01221. 
Schade \& Sanabria et al. NKA/Src in NASH progression

65. Magne F, Gotteland M, Gauthier L, et al. The Firmicutes/Bacteroidetes Ratio: A Relevant Marker of Gut Dysbiosis in Obese Patients? Nutrients 2020;12(5). DOI:

10.3390/nu12051474.

66. Greenhill C. Gut microbiota: Firmicutes and Bacteroidetes involved in insulin resistance by mediating levels of glucagon-like peptide 1. Nat Rev Endocrinol 2015;11(5):254. DOI: 10.1038/nrendo.2015.40.

67. Chen YH, Chiu CC, Hung SW, et al. Gnotobiotic mice inoculated with Firmicutes, but not Bacteroidetes, deteriorate nonalcoholic fatty liver disease severity by modulating hepatic lipid metabolism. Nutr Res 2019;69:20-29. DOI: 10.1016/j.nutres.2019.07.001.

68. Mouzaki M, Comelli EM, Arendt BM, et al. Intestinal microbiota in patients with nonalcoholic fatty liver disease. Hepatology 2013;58(1):120-7. DOI: 10.1002/hep.26319.

69. Mendez-Salazar EO, Ortiz-Lopez MG, Granados-Silvestre MLA, Palacios-Gonzalez B, Menjivar M. Altered Gut Microbiota and Compositional Changes in Firmicutes and Proteobacteria in Mexican Undernourished and Obese Children. Front Microbiol 2018;9:2494. DOI: 10.3389/fmicb.2018.02494.

70. Jiang W, Wu N, Wang $\mathrm{X}$, et al. Dysbiosis gut microbiota associated with inflammation and impaired mucosal immune function in intestine of humans with non-alcoholic fatty liver disease. Sci Rep 2015;5:8096. DOI: 10.1038/srep08096.

71. Ma C, Kesarwala AH, Eggert $\mathrm{T}$, et al. NAFLD causes selective CD4(+) T lymphocyte loss and promotes hepatocarcinogenesis. Nature 2016;531(7593):253-7. DOI: 10.1038/nature16969.

72. Herbein G, Doyle AG, Montaner LJ, Gordon S. Lipopolysaccharide (LPS) downregulates CD4 expression in primary human macrophages through induction of endogenous tumour necrosis factor (TNF) and IL-1 beta. Clin Exp Immunol 1995;102(2):430-7. DOI: 10.1111/j.1365-2249.1995.tb03801.x.

73. Nielsen JS, Larsson A, Ledet T, Turina M, Tonnesen E, Krog J. Rough-Form Lipopolysaccharide Increases Apoptosis in Human CD4(+) and CD8(+) T Lymphocytes. Scand J Immunol 2012;75(2):193-202. DOI: 10.1111/j.1365-3083.2011.02613.x.

74. Tariq Z, Green CJ, Hodson L. Are oxidative stress mechanisms the common denominator in the progression from hepatic steatosis towards non-alcoholic steatohepatitis (NASH)? Liver Int 2014;34(7):e180-90. DOI: 10.1111/liv.12523.

75. Rivera CA, Adegboyega $\mathrm{P}$, van Rooijen N, Tagalicud A, Allman M, Wallace M. Toll-like receptor-4 signaling and Kupffer cells play pivotal roles in the pathogenesis of nonalcoholic steatohepatitis. J Hepatol 2007;47(4):571-9. DOI: 10.1016/j.jhep.2007.04.019.

76. Siebler J, Galle PR, Weber MM. The gut-liver-axis: endotoxemia, inflammation, insulin resistance and NASH. J Hepatol 2008;48(6):1032-4. DOI: 10.1016/j.jhep.2008.03.007.

77. Kudo H, Takahara T, Yata Y, Kawai K, Zhang W, Sugiyama T. Lipopolysaccharide triggered TNF-alpha-induced hepatocyte apoptosis in a murine non-alcoholic steatohepatitis model. J Hepatol 2009;51(1):168-75. DOI: 10.1016/j.jhep.2009.02.032.

78. Tonan T, Fujimoto K, Qayyum A, et al. CD14 expression and Kupffer cell dysfunction in non-alcoholic steatohepatitis: superparamagnetic iron oxide-magnetic resonance image and pathologic correlation. J Gastroenterol Hepatol 2012;27(4):789-96. DOI: 10.1111/j.1440-1746.2011.07057.x.

79. Ogawa Y, Imajo K, Yoneda M, et al. Soluble CD14 levels reflect liver inflammation in patients with nonalcoholic steatohepatitis. PLoS One 2013;8(6):e65211. DOI: 10.1371/journal.pone.0065211.

80. Liu S, Khemlani LS, Shapiro RA, et al. Expression of CD14 by hepatocytes: upregulation by cytokines during endotoxemia. Infect Immun 1998;66(11):5089-98. DOI:

10.1128/IAI.66.11.5089-5098.1998. 
Schade \& Sanabria et al. NKA/Src in NASH progression

81. Su GL, Dorko K, Strom SC, Nussler AK, Wang SC. CD14 expression and production by human hepatocytes. J Hepatol 1999;31(3):435-42. DOI: 10.1016/s0168-8278(99)800348.

82. Luo $\mathrm{P}$, Yin $\mathrm{P}$, Hua $\mathrm{R}$, et al. A Large-scale, multicenter serum metabolite biomarker identification study for the early detection of hepatocellular carcinoma. Hepatology 2017. DOI: 10.1002/hep.29561.

83. Gall WE, Beebe K, Lawton KA, et al. alpha-hydroxybutyrate is an early biomarker of insulin resistance and glucose intolerance in a nondiabetic population. PLoS One 2010;5(5):e10883. DOI: 10.1371/journal.pone.0010883.

84. Aon MA, Bhatt N, Cortassa SC. Mitochondrial and cellular mechanisms for managing lipid excess. Front Physiol 2014;5:282. DOI: 10.3389/fphys.2014.00282.

85. Aravinthan A, Challis B, Shannon N, Hoare M, Heaney J, Alexander GJ. Selective insulin resistance in hepatocyte senescence. Exp Cell Res 2015;331(1):38-45. DOI: 10.1016/j.yexcr.2014.09.025.

86. Feldstein AE, Wieckowska A, Lopez AR, Liu YC, Zein NN, McCullough AJ. Cytokeratin18 fragment levels as noninvasive biomarkers for nonalcoholic steatohepatitis: a multicenter validation study. Hepatology 2009;50(4):1072-8. DOI: 10.1002/hep.23050.

87. Li Z, Cai T, Tian J, et al. NaKtide, a Na/K-ATPase-derived peptide Src inhibitor, antagonizes ouabain-activated signal transduction in cultured cells. J Biol Chem 2009;284(31):21066-76. DOI: 10.1074/jbc.M109.013821.

88. Pratt R, Lakhani HV, Zehra M, Desauguste R, Pillai SS, Sodhi K. Mechanistic Insight of $\mathrm{Na} / \mathrm{K}-\mathrm{ATP}$ ase Signaling and HO-1 into Models of Obesity and Nonalcoholic Steatohepatitis. Int J Mol Sci 2019;21(1). DOI: 10.3390/ijms21010087.

89. Yan Y, Shapiro AP, Haller S, et al. Involvement of reactive oxygen species in a feedforward mechanism of $\mathrm{Na} / \mathrm{K}-\mathrm{ATPase}-$ mediated signaling transduction. J Biol Chem 2013;288(47):34249-58. DOI: 10.1074/jbc.M113.461020. 
Schade \& Sanabria et al. NKA/Src in NASH progression

\section{Figure Legends}

Figure 1. Variations on microbiota communities in a NASH murine model. A) The proportion of Bacteroidetes was consistently decreased in the HFD vs. the NMC group at 12, 24 and 48 weeks. In contrast, a significantly greater proportion of Verrucomicrobia was found in the HFD group at 24 and 48 weeks. B) The proportion of Bacteroidetes and Firmicutes was decreased and the proportion of Verrucomicrobia was increased in the HFD group when compared to NMC. Most notably, the pNaK group demonstrated a microbial profile almost identical to NMC.

Figure 2. A) Gut $C D 4^{+} \& C D 8^{+}$Populations at 24 weeks. A significant decrease in $\mathrm{CD} 4^{+}$cells was observed in the pNaKtide treated group when compared to NMC, while a significant decrease in $\mathrm{CD}^{+}$cells in pNaKtide and Exercise groups was observed compared to NMC (Figure 3, $\mathrm{p}<0.05$, t-test). The combined population of $\mathrm{CD} 4^{+} \& \mathrm{CD} 8^{+}$cells followed a similar trend of being significantly decreased in pNaKtide and Exercise compared to NMC. B) Liver CD14 Population at 24 weeks. Liver CD14 ${ }^{+}$cells were significantly increased in the pNaKtide and Exercise groups compared to NMC (Figure 4, $\mathrm{p}<0.05$, t-test)

Figure 3. Non-targeted Metabolic prints signatures on treated plasma from diet-induced NASH in the rodent. $\boldsymbol{A})$ Metabolites prints separated experimental groups by diet HFD vs NMC and by intervention (no intervention vs. pNaKtide/exercise, respectively, $\mathrm{p}<0.01$ by PCA). B) Metabolic prints displayed as Heat Maps. Metabolite data was $\log ^{10}$ transformed and displayed as a visual array NMC vs. HFD groups, NMC vs. HFD+pNaKtide groups, and NMC vs. HFD+Exercise groups. There were significant differences in the metabolic print among groups $(p<0.01, P C A)$.

Figure 4. Total body compartments, morphology, glucose, and glutathione sp. in the Murine model of NASH. A) Rodents exposed to HFD significantly increased their total body weight (TBW) mainly due to an expansion of their fat mass compartment when compared to the NMC group ( $p<0.05$, by ANOVA followed by t-test). B) There was a significant increase in the NAS score of all HFD when compared to the NMC group $\left(p<0.01\right.$, by $\left.x^{2}\right)$. In addition, there was a significant increase in the fibrosis score as well as in the apoptotic and senescence activities in the HFD groups by week 24 when compared to the NMC group $(p<0.01)$. C) Glucose concentration from the NMC group was significantly different when compared to the HFD by week 24 ( $p<0.05)$. Animals in the HFD group had a significantly higher concentration of GSH, and GS:SG when compared to the NMC at week $24(p<0.01)$.

Figure 5. Total body compartments, morphology, glucose, and glutathione sp. in the Murine model of NASH by treatment. A) Rodents exposed to HFD increased their total body weight (TBW) mainly due to an expansion of their fat mass with similar values among all groups ( $p>0.05$, by ANOVA). B) There was a significant increase in the NAS score in the HFD group when compared to $p N a K t i d e$ or Exercise groups $\left(p<0.05\right.$, by $\left.x^{2}\right)$. In addition, there was a significant decrease in the fibrosis score in the pNaKtide and exercise groups $(p<0.05)$. A

dramatic difference was observed in the number of apoptotic and cell senescence activities. A significant decrease in apoptosis was observed in the HFD group when compared to pNaKtide and Exercise groups by week 24 ( $p<0.01)$. In contrast, cell senescence was significantly increase in the exercise group compared to the HD or pNaKtide groups $(p<0.05)$. C) Glucose concentration from the HFD group was significantly different when compared to the pNaKtide or 
Schade \& Sanabria et al. NKA/Src in NASH progression

exercise groups by week 24 ( $p<0.05$ by ANOVA). Animals in the HFD group had a significantly higher concentration of GSH, and GS:SG when compared to the pNaKtide and Exercise groups at week 24 ( $p<0.01$, ANOVA).

Figure 6. Metabolic mediators of the NASH response and Liver Na/K-ATPase activity and expression. A) PGC1 $\alpha$ had a significant peak at week 16 in the pNaKtide and exercise groups when compared to HFD group with a second significant peak of PGC1 $\alpha$ in the exercise group at week 24 ( $p<0.01$ by ANOVA/ t-test). B) PPARy had similar behavior observed with the coactivator PGC1 $\alpha$ at weeks 16. C) There was a significant peak on Src-phosphorylation in the HDF group when compared to the pNaKtide by week 20 ( $p<0.05)$. D) FOXO1 expression was significantly up-regulated in the pNaKtide group when compared to the HFD group at week 24 $(p<0.01)$. E) There was a significant difference in the activity of the $\alpha 1-\mathrm{Na} / \mathrm{K}-\mathrm{ATPase}$ among groups at week 24 ( $p<0.05$, by ANOVA/ t-test). Protein activity from the livers of animals in the pNaKtide group was significantly higher when compared to the HFD or exercise groups and showed a similar activity when compared to the NMC group. Nevertheless, there was not a significant difference in the expression of the a1 subunit of the $\mathrm{Na} / \mathrm{K}-\mathrm{ATPase}$ among groups at week 24 ( $p>0.05$, ANOVA).

\section{APPENDIX}

Appendix Figure Legends

Figure A1. Experimental design and animal flow for the microbiota community and metabolic assessment on the Murine Model of diet-induced NASH. Female C57/BL6J rodents 7weeks old were exposed to normal mouse chow ( $\underline{\mathrm{NMC}}$ ), high fat diet + fructose ($\underline{\mathrm{HFD}}$ ) from 12 weeks to 48 weeks. A subset of animals was exposed to treatment protocols ( $\mathrm{pNaKtide}$ or exercise) for 12 weeks.

Figure A2. NASH Activity Score in the Murine Liver exposed to HFD plus interventions at 24 weeks. There was a significant increase in the macro-vesicular content on liver cells in the HFD groups with a peak at week 24 when compared with NMC and the interventions groups $(p<0.05$ by $\mathrm{X}^{2}$ ). Nevertheless, the storage of fat in liver cells as micro-vesicles in the NMC differed significantly from the HFD and pNaKtide groups $(p<0.05)$ but did not have a significant variation when compared to the Exercise group ( $p>0.05$ ). All HFD groups had a significant higher cell size where animals were exposed to HFD vs NMC, but similar among all HFD exposed groups $(p<0.05)$. In contrast, the inflammatory component was significantly larger in the HFD groups; it peaked at week 24 when compared to NMC and intervention groups $\left(p<0.05\right.$ by $\left.x^{2}\right)$. Interestingly, the liver inflammation in the intervention groups was reduced by week 16 and resolved by week 24 .

Figure A3. Morphological assessment of livers from diet-induced NASH in the rodent from week 0 to week 24. A) Liver NAFLD Activity Score (NAS) was determined by grading five images at 40x magnification on liver slides H\&E stained. Grading was carried out as described; B) Liver fibrosis was determined by grading five images at 40x magnification of Trichrome stained liver slides on a categorical scale by the fibrosis score;inflammatory cell infiltrate and cellular hypertrophy; C) Liver apoptotic activity was determined through grading five images at 40x magnification of labeling (TUNEL) stained liver slides, and D) liver cellular senescence was determined by grading five images at 40x magnification of liver slides stained for $\beta$ Galactosidase activity. 
Schade \& Sanabria et al. NKA/Src in NASH progression

Figure A4. Comparative metabolomic heat map signatures on treated plasma from diet-induced $\mathrm{NASH}$ in the rodent from week 0 to week 24. A) The NMC group array was displayed at week 0 (WONMC) and then the NMC from each week was compared to the HFD group from each week (weeks 12, 16, 20 and 24 and labeled W12HFD, W16HFD, W20HFD, and W24HFD, respectively); B) the NMC group array was displayed at week 0 (WONMC) and then the NMC from each week was compared to the pNaKtide group from each week at weeks 12, 16, 20 and 24 and labeled W12HFD, W16HFDP, W20HFDP, and W24HFDP, respectively, and C) the NMC group array was displayed at week 0 (WONMC). Then the NMC from each week was compared to the Exercise group from each week (at weeks 12, 16, 20, and 24 and labeled W12HFD, W16HFDE, W20HFDE, and W24HFDE, respectively). The program compared each metabolite for each animal among groups and displayed a black color on similar value, a magenta color for a significantly higher concentration, and a yellow color for a significantly lower concentration of the metabolites. In the $\mathrm{Y}$-axis, the metabolite variable was entered while in the $\mathrm{X}$-axis animal/group was recorded. Because of the Log transformation, differences are by a 100 to a 1000 factor between groups. There was a significant difference in both the metabolic print among groups at weeks 16,20 , and 24 and the progression of the metabolic print for each group $(\mathrm{p}<0.0$, by PCA).

Figure A5. Homo-Oxygenase-1 (HO-1) expression and assessment of pNaKtide presence on murine livers with $\mathrm{NASH}$. A) Liver tissue was homogenized from experimental groups, and the expression of HO-1 and presence of pNaKtide was interrogated by WB methods at 24W. There was a significantly lower expression of HO-1 in the liver from animals exposed to NMC when compared to animals exposed to HFD or, Exercise ( $p<0.05$, by ANOVA). HO- 1 expression among HFD groups was similar ( $p>0.05)$. B) While liver tissue from the NMC group served as negative control, pNaKtide ( $95 \%$ pure peptide) served as positive control. Actin served as the house-keeping protein. pNaK was present in all livers from animals exposed to IP pNaK. 